\title{
Optimal Risk-Sharing Across a Network of Insurance Companies
}

\author{
Ettlin, Nicolas ; Farkas, Walter ; Kull, Andreas ; Smirnow, Alexander
}

\begin{abstract}
Risk transfer is a key risk and capital management tool for insurance companies. Transferring risk between insurers is used to mitigate risk and manage capital requirements. We investigate risk transfer in the context of a network environment of insurers and consider capital costs and capital constraints at the level of individual insurance companies. We demonstrate that the optimisation of profitability across the network can be achieved through risk transfer. Considering only individual insurance companies, there is no unique optimal solution and, a priori, it is not clear which solutions are fair. However, from a network perspective, we derive a unique fair solution in the sense of cooperative game theory. Implications for systemic risk are briefly discussed.
\end{abstract}

DOI: https://doi.org/10.1016/j.insmatheco.2020.08.002

Posted at the Zurich Open Repository and Archive, University of Zurich

ZORA URL: https://doi.org/10.5167/uzh-190721

Journal Article

Published Version

Originally published at:

Ettlin, Nicolas; Farkas, Walter; Kull, Andreas; Smirnow, Alexander (2020). Optimal Risk-Sharing Across a Network of Insurance Companies. Insurance: Mathematics and Economics, 95:39-47.

DOI: https://doi.org/10.1016/j.insmatheco.2020.08.002 


\section{Swiss Finance Institute Research Paper Series $\mathrm{N}^{\circ} 20-52$}

Optimal Risk-Sharing Across a Network of Insurance Companies

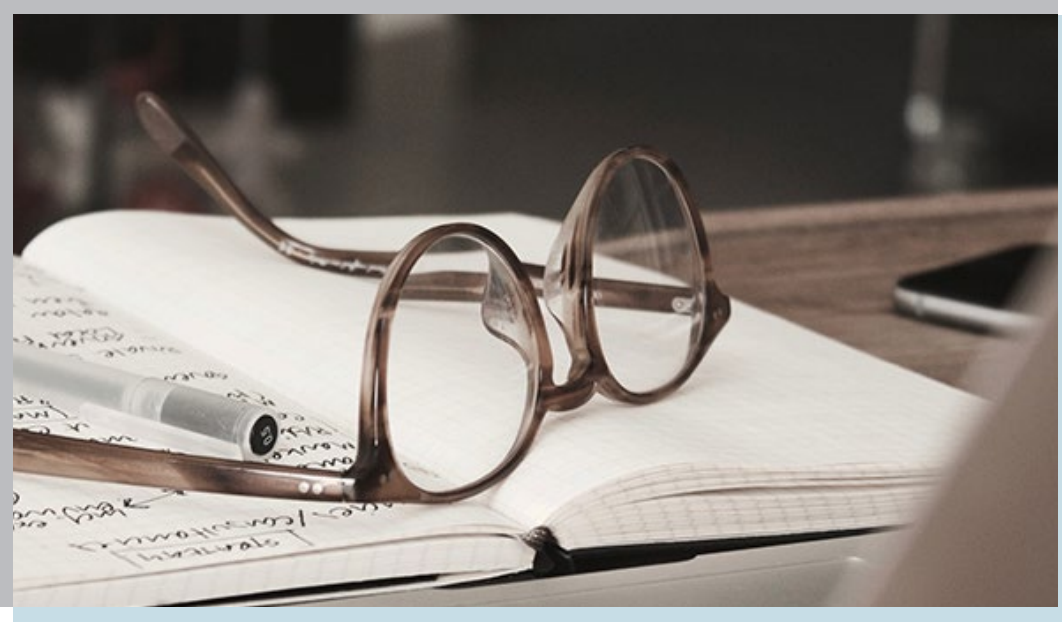

Nicolas Ettlin

University of Basel

Walter Farkas

University of Zurich, ETH Zurich, and Swiss Finance Institute

Andreas Kull

University of Basel and BerninaRe Ltd.

Alexander Smirnow

University of Zurich 


\title{
Optimal risk-sharing across a network of insurance companies
}

\author{
Nicolas Ettlin ${ }^{\dagger} \quad$ Walter Farkas ${ }^{\S *} \quad$ Andreas Kull ${ }^{\dagger \ddagger}$ \\ Alexander Smirnow ${ }^{\S}$
}

June, 2020

\begin{abstract}
Risk transfer is a key risk and capital management tool for insurance companies. Transferring risk between insurers is used to mitigate risk and manage capital requirements. We investigate risk transfer in the context of a network environment of insurers and consider capital costs and capital constraints at the level of individual insurance companies. We demonstrate that the optimisation of profitability across the network can be achieved through risk transfer. Considering only individual insurance companies, there is no unique optimal solution and, a priori, it is not clear which solutions are fair. However, from a network perspective, we derive a unique fair solution in the sense of cooperative game theory. Implications for systemic risk are briefly discussed.
\end{abstract}

Keywords risk transfer, risk-based capital, reinsurance, return optimisation, conditional expected shortfall

JEL classification G13, G22, D85, C57, C71

\section{Introduction}

Insurance companies can transfer their risk to other entities, for example reinsurance companies, to mitigate loss potential and reduce the cost of capital. For a single insurance company optimising risk transfer is straightforward as it means to compare the price for risk transfer to its benefits in terms of mitigated losses and reduced cost of risk capital. For a network of insurers, however, the optimisation can take different perspectives and therefore the meaning of optimising risk transfer in a network is not clear. In this article, we investigate risk transfer in the context of a network of insurance companies. We use a general framework with passive and active reinsurance, that means insurers can transfer their risk to reinsurance companies and also between themselves. We take an economic point of view and consider capital costs and capital constraints

\footnotetext{
*ETH Zürich, Department of Mathematics, Rämistrasse 101, 8092 Zürich, Switzerland.

${ }^{\dagger}$ University of Basel, Department of Mathematics and Computer Science, Spiegelgasse 1, CH-4051 Basel, Switzerland, emails: nicolas.ettlin@stud.unibas.ch and andreas.kull@unibas.ch.

${ }^{\ddagger}$ Bernina Re Ltd., Victoria Place, 31 Victoria Street, Hamilton HM10, Bermuda.

${ }^{\S}$ University of Zurich, Department of Banking and Finance, Plattenstrasse 32, CH-8032 Zurich, Switzerland, email: alexander.smirnow@bf.uzh.ch, corresponding author.
} 
at the level of individual insurers. A key result is that while there is no unique solution optimising risk transfer for all insurance companies at the individual level, there exists a unique, fair and optimal solution in the sense of cooperative game theory if the network perspective is adopted.

Optimal risk sharing between insurance and reinsurance companies has been considered by various authors. Research either takes the perspective of individual entities $[10,17$, $7]$ or, more recently, insurance groups $[21,18,23,15,5]$. Because of the ownership structure and related transparency of risk exposures, an optimisation of risk transfer for insurance groups can start from the comprehensive group standpoint. Related research results are not only relevant for the economics of insurance groups but also for insurance supervision and regulation. Indeed, recent findings point towards a significant impact of regulatory frameworks on optimising risk transfer and systemic risk as for example discussed by A. Asimit, A. Badescu, and A. Tsanakas [4]. Our starting point in this paper differs in the sense that we do not assume a group structure but consider risk transfer across a network of insurers. Optimal risk transfer thus is not considered from an overarching insurance group perspective but from the perspective of a network of individual insurers and game theory.

Risk transfer across a network of insurance companies has been considered by other authors as well. For example, A.-M. Hamm, T. Knispel, and S. Weber [16] show that risk transfer can significantly decrease overall capital requirements when using Value at Risk based risk measures. Other approaches aim to minimise the aggregated risk in a system by redistributing its components across participating agents, see for example the work of F.-B. Liebrich and G. Svindland [22] for an acceptance set based approach. A utility based framework has been suggested by F. Biagini, A. Doldi, J.-P. Fouque, M. Frittelli, and T. Meyer-Brandis [6].

The paper builds in part on the work of N. Ettlin [14] and generalises results on optimisation of risk transfer for two insurers as discussed by A. Kull [20] to multiple insurers. Furthermore, it draws a connection to cooperative game theory and related concepts of capital allocation as developed by M. Denault [12].

The paper is organised as follows. In Section 2, we briefly review monetary risk measures and define the Conditional Expected Shortfall. Furthermore, we explain the notion of risk capital, capital costs, capital allocation, risk aggregation, and related economics. In Section 3, we draw links between these concepts and risk transfer by introducing a network of insurance companies. We define the optimisation of risk transfer for individual insurance companies and the network in Section 4. We discuss that there is no unique optimal solution without further constraints. However, using principles from cooperative game theory we can identify a unique and in that sense fair solution to the optimisation of risk transfer. The paper concludes with a discussion of implications for insurance groups and the systemic risk debate in Section 6 .

\section{Economics of risk transfer}

The inherent uncertainty of the future as it manifests itself, for example in uncertain loss experience, is usually referred to as risk. Mathematically, the impact of the uncertainty of the future is described by random variables. To this end, let $(\Omega, \mathcal{F}, \mathbb{P})$ denote a probability space. Let $\mathcal{X}$ be some real topological vector space equipped with an 
appropriate preorder $\leq$. For us $\mathcal{X}$ is a subset of $L^{0}(\Omega, \mathcal{F}, \mathbb{P})$, the space of all equivalence classes with respect to $\mathbb{P}$-a.s. equality of measurable maps from $(\Omega, \mathcal{F})$ to $\mathbb{R}$ with its Borel $\sigma$-algebra. Since we will work with Expected Shortfall, we will usually choose $\mathcal{X}=L^{p}(\Omega, \mathcal{F}, \mathbb{P})$, for $p \in[1, \infty]$. Furthermore, we will work with continuous random variables.

\subsection{Measuring risk}

Risk measures quantify the risk of a financial position. They are functionals $\rho: \mathcal{X} \rightarrow \mathbb{R}$ with $X \mapsto \rho(X)$. P. Artzner, F. Delbaen, J. Eber and D. Heath [3] introduce four axioms a practicable risk measure should fulfil and define what is known as coherent risk measures on the space of bounded random variables. F. Delbaen [11] extends the concept of coherent risk measures to general probability spaces. We will adapt the framework of A. J. McNeil, R. Frey, and P. Embrechts [24] and define risk measures on random variables which represent losses. This means that for our risk measures losses are positive. Notice that therefore the four coherency axioms differ slightly from the ones in [3]. In particular, instead of decreasing monotonicity we have increasing monotonicity, and for $L \in \mathcal{X}, \ell \in \mathbb{R}$ the translation property reads $\rho(L+\ell)=\rho(L)+\ell$.

In the following, we will use the (right-tail) Expected Shortfall or Tail Value at Risk as the coherent risk measure. ${ }^{1}$ For a loss $L$ with $\mathbb{E}[|L|]<\infty$ the Expected Shortfall at a confidence level $\alpha \in(0,1)$ is defined as

$$
\operatorname{ES}_{\alpha}(L)=\frac{1}{1-\alpha} \int_{\alpha}^{1} \operatorname{VaR}_{\beta}(L) \mathrm{d} \beta
$$

where $\operatorname{VaR}_{\beta}: \mathcal{X} \rightarrow \mathbb{R}$ denotes the Value at Risk at a probability level $\beta$. The Value at Risk of a loss random variable $L$ with continuous and strictly increasing cumulative distribution function $F_{L}: \mathbb{R} \rightarrow[0,1]$ is given by its inverse, $\operatorname{VaR}_{\alpha}(L)=F_{L}^{-1}(\alpha){ }^{2}$ It can be shown, see for example [24, Lemma 2.16], that in this case, the Expected Shortfall coincides with the Conditional Value at Risk, which is defined as the expectation of $L$ conditioned on that $L$ exceeds its Value at Risk at level $\alpha$,

$$
\operatorname{ES}_{\alpha}(L)=\operatorname{CVaR}_{\alpha}(L)=\mathbb{E}\left[L \mid L \geq \operatorname{VaR}_{\alpha}(L)\right] .
$$

Notice that in general, this equation does not hold for discontinuous loss distributions as described in [24, Remark 2.17] and proved by C. Acerbi and D. Tasche [1] in Proposition 3.2. We will use this equality to draw a connection to the related conditional risk measure termed Conditional Expected Shortfall $\mathrm{CES}_{\alpha, Y}$. It is defined as the expectation of $L$ conditioned on another loss random variable $Y$ at a probability level $\alpha$,

$$
\operatorname{CES}_{\alpha, Y}(L)=\mathbb{E}\left[L \mid Y \geq \operatorname{VaR}_{\alpha}(Y)\right]
$$

This conditional risk measure is favourable due to its properties, in particular, its additivity which allows for an additive and consistent allocation of risk and capital. A wider context is given by G. Venter [27].

\footnotetext{
${ }^{1}$ For a proof that the Expected Shortfall is indeed coherent see for example [24, Proposition 6.9].

${ }^{2}$ For more details see for example [24, Definition 2.10, Definition 2.12].
} 


\subsection{Risk capital and capital costs}

For insurance companies capital provides protection against insolvency or ruin due to large unexpected losses. Insurers hold certain amounts of capital, termed riskbased capital, in order to ensure the ability to sustain significant, unexpected losses. Determining the risk-based capital for a specific risk is usually done by applying risk measures. For instance, the supervisory framework of Solvency II prescribes the usage of Value at Risk and the Swiss Solvency Test of Expected Shortfall as risk measures for calculating regulatory capital requirements.

The exact definition of risk-based capital and the different ways to calculate it is a widely discussed topic. Definitions depend on scopes ranging from regulatory aspects to company-internal capital management and performance measurement. Considering a portfolio with underwriting profits reflected by the random variable $X \in \mathcal{X}$ and a coherent risk measure $\rho$, the risk-based capital corresponding to the portfolio $X$ is given by

$$
\operatorname{RBC}(X)=\rho(-X)
$$

Notice that since $X$ represents returns, we use $-X$ inside the risk measure. Holding capital comes at a cost proportional to the amount of risk-based capital,

$$
C(X)=\eta \operatorname{RBC}(X)
$$

where the cost of capital rate $\eta \in(0,1)$ depends on various factors. These factors include the capital structure of the company, the type and riskiness of the business, and other specificities. The cost of capital rate $\eta$ for an insurer typically lies in the range of $4 \%$ to $15 \%$, see for example the cost of capital study by KPMG [19]. Any meaningful economic analysis of the economics of insurance and risk transfer should take this cost of capital into account.

We consider now the case of a simplified non-life insurer that underwrites short-tail business over a single time period. Neglecting the cost of capital, the net underwriting profit is given by

$$
U=P-L,
$$

where $P$ stands for the premium income and $L$ for the accumulated gross loss amount. To keep things simple, consider the premium $P \geq 0$ to be a constant. The underwriting profit $Z$, after including the cost of capital, is defined as

$$
Z=U-C(Z)=U-\eta \operatorname{RBC}(Z)=U-\eta \rho(-Z) .
$$

Here, $\operatorname{RBC}(Z)$ stands for the amount of capital allocated to support the risk related to $Z$.

In general, insurance companies hold risk-based capital only up to a probability corresponding to a company-specific risk appetite level $\alpha$ or regulatory requirements and not to the full range of potential adverse results. For example, the Swiss Solvency Test prescribes $\alpha=0.99$. Furthermore, the determination of the risk-based capital $\operatorname{RBC}(Z)$ also depends on the situation relative to which it is defined. 
Lemma 2.1. The recursive formula for $Z$ in Equation (2.1) can be explicitly stated as

$$
Z=U-\frac{\eta}{1-\eta} \rho(-U) .
$$

Proof. Inserting $Z$ itself in Equation (2.1) and using the translation property of $\rho$, we get the identity $Z=U-\eta \rho(-U)-\eta^{2} \rho(-Z)$. Writing $U=\eta U+(1-\eta) U$ and then using Equation (2.1) again, we arrive at

$$
Z=(1-\eta) U-\eta \rho(-U)+\eta(U-\eta \rho(-Z))=(1-\eta) U-\eta \rho(-U)+\eta Z .
$$

Solving for $Z$ concludes the proof.

Hence, to calculate the risk-based capital of $Z$ we apply the risk measure to $-Z$ and use the translation property to get

$$
\rho(-Z)=\rho(-U)\left(1+\frac{\eta}{1-\eta}\right)=\frac{\rho(-U)}{1-\eta} .
$$

As mentioned before, we choose the Expected Shortfall as our risk measure so that

$$
\operatorname{RBC}(Z)=\frac{\operatorname{ES}_{\alpha}(-U)}{1-\eta}
$$

We will assume capital costs $\eta$ and risk appetite levels $\alpha$ to be fixed. Both of these assumptions are significant simplifications which, however, can be motivated by similar regulatory constraints.

Remark 2.2. Notice that we can use the recursive formula in Equation (2.1) directly to calculate the risk-based capital of $Z$ as

$$
\rho(-Z)=\rho(-U+\eta \rho(-Z))=\rho(-U)+\eta \rho(-Z) .
$$

Solving this with respect to $\rho(-Z)$, we arrive at Equation (2.2).

\subsection{Capital allocation and capital aggregation}

For various purposes ranging from management structure to risk and performance measurement, insurance companies group their business into sub-portfolios consisting of business of similar risk characteristics, known as "lines of business". For company-level risk and performance management the quantification of the contribution of risk-based capital at the sub-portfolio level to the overall risk capital, or vice versa, the allocation of overall risk-based capital to a sub-portfolio, is fundamental. For a review of capital allocation principles see for example [27] and the references therein.

Consider the case of a simplified non-life insurer. We want to split its business with underwriting profit $Z$ given in (2.1) into $n \in \mathbb{N}$ business lines. For $i \in\{1, \ldots, n\}$ their underwriting profit should be given by

$$
Z_{i}=U_{i}-\eta \mathrm{RBC}_{Z}\left(Z_{i}\right)
$$


where $U=\sum_{i=1}^{n} U_{i}$ and $\mathrm{RBC}_{Z}\left(Z_{i}\right)$ stands for the allocated risk-based capital of $Z_{i}$, but with respect to the total economic profit $Z$. Here, we explicitly choose the Conditional Expected Shortfall,

$$
\operatorname{RBC}_{Z}\left(Z_{i}\right)=\mathrm{CES}_{\alpha,-Z}\left(-Z_{i}\right)
$$

So the risk-based capital of $Z_{i}$ is allocated dependent on its contribution to the overall risk. However, to ensure that we have indeed $Z=\sum_{i=1}^{n} Z_{i}$, we must have $\operatorname{RBC}(Z)=$ $\sum_{i=1}^{n} \mathrm{RBC}_{Z}\left(Z_{i}\right)$. The two lemmas in Appendix A.1 show that the Conditional Expected Shortfall is indeed consistent with our requirements.

In a strict sense, allocating capital is only meaningful for risks that are pooled in a single portfolio, or on the balance sheet of a single insurer. Only in this case, diversification effects materialise economically through reducing capital costs. If individual insurance companies are considered which operate on a standalone basis, then diversification may be present in the risk they underwrite. However, from an economic perspective this diversification may not be realised as capital costs are incurred at the level of individual insurers. Thus, rather than considering an individual insurer we consider a network of insurers each determining its risk-based capital $\operatorname{RBC}\left(Z_{i}\right)$ on a standalone basis. Assuming the coherence of the underlying risk measure, subadditivity implies

$$
\mathrm{RBC}_{\text {Market }}:=\operatorname{RBC}\left(\sum_{i=1}^{n} Z_{i}\right) \leq \sum_{i=1}^{n} \operatorname{RBC}\left(Z_{i}\right)=: \mathrm{RBC}_{\text {Network }}
$$

where $\mathrm{RBC}_{\text {Market }}$ denotes the risk-based capital for a situation in which all risks are pooled, the market portfolio, and $\mathrm{RBC}_{\text {Network }}$ stands for the sum of standalone riskbased capital. The inequality in (2.6) implies that the aggregated standalone capital cannot be less than the risk-based capital $\mathrm{RBC}_{\text {Market }}$ for fully pooled risks. The difference $\mathrm{RBC}_{\text {Network }}-\mathrm{RBC}_{\text {Market }}$ is non-negative and it is related to the diversification achieved by the network of insurance companies relative to the overall market portfolio. The higher the diversification achieved by the insurers in the network, the lower this difference. Full diversification relative to the market portfolio is reached if $\mathrm{RBC}_{\text {Network }}-\mathrm{RBC}_{\text {Market }}=0$.

Next, we consider the profitability distributions at the market level,

$$
Z_{\text {Market }}=\sum_{i=1}^{n}\left(P_{i}-L_{i}\right)-\eta \operatorname{RBC}\left(\sum_{i=1}^{n} Z_{i}\right)=\sum_{i=1}^{n}\left(P_{i}-L_{i}\right)-\eta \mathrm{RBC}_{\text {Market }},
$$

and at the level of aggregated standalone profitability,

$$
Z_{\text {Network }}=\sum_{i=1}^{n} P_{i}-L_{i}-\eta \operatorname{RBC}\left(Z_{i}\right)=\sum_{i=1}^{n}\left(P_{i}-L_{i}\right)-\eta \mathrm{RBC}_{\text {Network }}
$$

In terms of expected profitability, Equation (2.6) implies that

$\mathbb{E}\left[Z_{\text {Market }}\right]=\sum_{i=1}^{n} \mathbb{E}\left[P_{i}-L_{i}\right]-\eta \mathrm{RBC}_{\text {Market }} \geq \sum_{i=1}^{n} \mathbb{E}\left[P_{i}-L_{i}-\eta \mathrm{RBC}\left(Z_{i}\right)\right]=\mathbb{E}\left[Z_{\text {Network }}\right]$ 
The difference between $\mathbb{E}\left[Z_{\text {Market }}\right]$ and $\mathbb{E}\left[Z_{\text {Network }}\right]$ is driven by the level of diversification achieved by the insurance companies which are part of the network and is equal to

$$
\mathbb{E}\left[Z_{\text {Market }}\right]-\mathbb{E}\left[Z_{\text {Network }}\right]=\eta\left(\mathrm{RBC}_{\text {Network }}-\mathrm{RBC}_{\text {Market }}\right) .
$$

This means that $\mathbb{E}\left[Z_{\text {Market }}\right]-\mathbb{E}\left[Z_{\text {Network }}\right]$ is the difference in the cost of capital for the fully diversified market portfolio and the sum of the standalone cost of capital at the level of individual insurance companies. The question then arises whether risk transfer between insurers can improve profitability at the level of individual insurers and the network as a whole. To answer this question, the economics of risk transfer needs to be clarified.

\section{Risk transfer across a network of insurers}

Pooling and sharing risk generally increases diversification and thus may reduce riskbased capital and related capital costs. Beyond risk management purposes and meeting capital constraints, transferring risk may also be driven by purely economic considerations as for an insurer the cost of transferring risk may be lower than the cost of keeping the risk on its balance sheet. Assuming an adequate premium for risk transfer is paid, a "win-win situation", whereby both parties increase their profitability, can occur. In essence, risk transfer will enable insurers to access and share the diversification inherent to the network.

\subsection{Risk transfer network}

Risk transfer usually takes place between an insurer and a reinsurer. We adopt the more general view that insurance companies can transfer risk between themselves. In practice, this situation occurs if insurance companies participate in passive reinsurance, that is, transferring risk to a reinsurer, active reinsurance, that is, accepting risk from another insurer, or in insurance groups which optimise capital requirements by internal risk transfer.

Including risk transfer into the model of the simplified non-life insurer introduced in Section 2.2, the underwriting profit after risk transfer is given by

$$
Z^{\star}=U-\eta \operatorname{RBC}\left(Z^{\star}\right)=P-L+R-P^{\star}-\eta \operatorname{RBC}\left(Z^{\star}\right),
$$

where $R$ represents the reinsurance recoveries due to risk transfer and $P^{\star}$ is the reinsurance premium paid for the risk transfer.

To structure the problem, we represent our network of insurers by a graph. The vertices of the graph represent the insurers and the edges represent their risk transfers. For each risk transfer related quantity we use two indices, $i$ and $j$, to indicate the direction of risk transfer. The first index denotes the risk assuming party and the second index the risk ceding party. For example, $P_{i j}^{\star}$ denotes the premium that insurer $j$ pays insurer $i$, whereas $R_{i j}$ denotes the recovery that insurer $i$ pays insurer $j$. See Figure 1 for an illustration. The arrows indicate the direction of the risk transfer. 


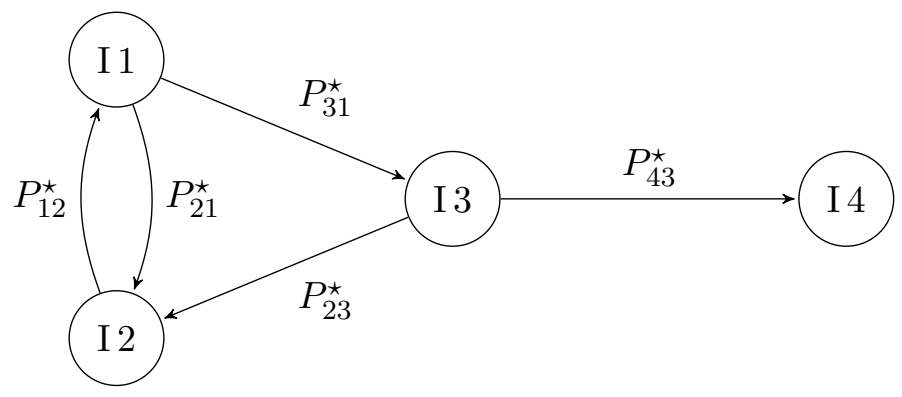

Figure 1: System of insurers and network of risk transfer.

\subsection{The price of transferring risk}

Principles defining the cost of risk transfer are generally referred to as premium principles. Most premium principles split the premium into the expected loss originating from risk transfer and the loading. Common loadings principles are, for example, the Expected Value principle or the Variance principle. For a review see for example C. Daykin, T. Pentikainen, and M. Pesonen [9] and for the broader context see M. Dacorogna [8]. In line with economic principles laid out in Section 2.2, we define the loading as the additional cost of capital incurred due to the risk transfer by the risk accepting party. Notice that by this definition, it is the risk accepting party which defines the price for risk transfer. This is a simplification as in reality, pricing of risk transfer is often negotiated by the risk transferring and risk accepting parties.

Following concepts discussed in Section 2.2 and Section 2.3, the net underwriting profit of the risk accepting insurer $i$ after risk transfer from insurer $j$ is

$$
Z_{i}^{\star}=Z_{i}+P_{i j}^{\star}-R_{i j}-\eta \mathrm{RBC}_{Z_{i}^{\star}}\left(P_{i j}^{\star}-R_{i j}\right) .
$$

The risk-based capital $\mathrm{RBC}_{Z_{i}^{\star}}$ allocated to the risk transfer from insurer $j$ to $i$ relative to the profit $Z_{i}^{\star}$ is defined as

$$
\mathrm{RBC}_{Z_{i}^{\star}}\left(P_{i j}^{\star}-R_{i j}\right)=\mathrm{CES}_{\alpha,-Z_{i}^{\star}}\left(R_{i j}-P_{i j}^{\star}\right) .
$$

Based on the discussion above and Equation (3.1), we define the premium for risk transfer as

$$
P_{i j}^{\star}=\mathbb{E}\left[R_{i j}\right]+\eta \mathrm{RBC}_{Z_{i}^{\star}}\left(P_{i j}^{\star}-R_{i j}\right) .
$$

This premium principle states that the premium $P_{i j}^{\star}$ for the risk transfer from insurer $j$ to insurer $i$ is equal to the expected loss due to paid recoveries $\mathbb{E}\left[R_{i j}\right]$ plus the loading $\eta \mathrm{RBC}_{Z_{i}^{\star}}\left(P_{i j}^{\star}-R_{i j}\right)$. The latter reflects the incurred capital costs for the risk accepting insurer $i$. Together with Equation (3.2) and the linearity of the Conditional Expected Shortfall this leads to

$$
P_{i j}^{\star}=\frac{1}{1+\eta}\left(\mathbb{E}\left[R_{i j}\right]+\eta \mathrm{CES}_{\alpha,-Z_{i}^{\star}}\left(R_{i j}\right)\right) .
$$




\subsection{Proportional risk transfer}

While risk transfer can take proportional and non-proportional forms, all relations in Section 3.2 are of general form. For the sake of simplicity and analytical tractability, we will consider the case of proportional risk transfer here. If non-proportionality is assumed, analytical expressions (Equation (3.4) to Equation (3.7)) for non-proportional risk transfer are not readily available. However, because of the universality of our approach, a numerical evaluation is feasible and it is left for later work. Here, parties transfer proportions of their risk exposures for an adequate premium. Proportional risk transfer can be characterised by one parameter, the proportion of risk that is ceded from one to another party or the related retention of risk that is kept.

To increase readability, we will omit the boundaries of the running variable when summing over insurers. So for some fixed $i \in\{1, \ldots, n\} \subset \mathbb{N}$, we define

$$
\sum_{j \neq i} a_{j}:=\sum_{j \in J_{i}} a_{j}
$$

where $J_{i}=\{j \in \mathbb{N} \mid 1 \leq j \leq n, j \neq i\}$.

For a network of $n \in \mathbb{N}$ insurers mutually transferring risk, the proportional risk transfer can be represented by an $(n \times n)$ - matrix $C \in[0,1]^{n \times n}$. For $i, j \in\{1, \ldots, n\}, i \neq j$, let $C_{i j}$ denote the portion of the risk $L_{j}$ insurer $j$ is transferring to insurer $i$, or equivalently, the proportion of risk $L_{j}$ the insurer $i$ is accepting from insurer $j$. Furthermore, set $C_{i i}=1-\sum_{j \neq i} C_{j i}$. Then the matrix becomes

$$
C=\left(\begin{array}{cccc}
1-\sum_{j \neq 1} C_{j 1} & C_{12} & \cdots & C_{1 n} \\
C_{21} & 1-\sum_{j \neq 2} C_{j 2} & & C_{2 n} \\
\vdots & & \ddots & \vdots \\
C_{n 1} & \cdots & & 1-\sum_{j \neq n} C_{j n}
\end{array}\right)
$$

Notice that the matrix $C$ satisfies the following properties.

- $R_{i j}(C)=C_{i j} L_{j}($ for $i \neq j$ ) corresponds to losses incurred by insurer $i$ due to risk transfer from insurer $j$ to insurer $i$.

- $R_{j i}(C)=C_{j i} L_{i}($ for $i \neq j$ ) corresponds to recoveries received by insurer $i$ due to risk transfer from insurer $i$ to insurer $j$.

- Elements $C_{i i}=1-\sum_{j \neq i} C_{j i}$ represent the retention levels for risk $L_{i}$ of insurer $i$. Assuming that no shorting of risk positions is possible, we have $\sum_{j \neq i} C_{j i} \leq 1$ and $C_{i i} \geq 0$.

- For each insurer $i$ we have $\sum_{j=1}^{n} C_{j i}=1$.

Notice that the matrix $C$ can be decomposed into the adjacency matrix characterising risk transfer where the diagonal elements are equal to zero, and the diagonal matrix representing risk retention.

The net underwriting profit of insurer $i \in\{1, \ldots, n\}$ taking into account all risk transfer 
is given by

$$
Z_{i}^{\star}(C)=P_{i}-L_{i}^{\star}(C)-\sum_{j \neq i}\left(P_{j i}^{\star}(C)-P_{i j}^{\star}(C)\right)-\eta \operatorname{RBC}\left(Z_{i}^{\star}(C)\right),
$$

where the total net loss $L_{i}^{\star}(C)$ incurred by insurer $i$ is the operational loss adjusted by the received and paid recoveries,

$$
\begin{aligned}
L_{i}^{\star}(C) & :=L_{i}-\sum_{j \neq i}\left(R_{j i}(C)-R_{i j}(C)\right)=L_{i}\left(1-\sum_{j \neq i} C_{j i}\right)+\sum_{j \neq i} C_{i j} L_{j} \\
& =L_{i} C_{i i}+\sum_{j \neq i} C_{i j} L_{j}=\sum_{j=1}^{n} C_{i j} L_{j} .
\end{aligned}
$$

$P_{i j}^{\star}(C)$ and $P_{j i}^{\star}(C)$ are defined as in Equation (3.3). In analogy to Remark 2.2, we derive the risk-based capital as

$$
\operatorname{RBC}\left(Z_{i}^{\star}(C)\right)=\frac{1}{1-\eta} \operatorname{ES}_{\alpha}\left(L_{i}^{\star}(C)-P_{i}+\sum_{j \neq i}\left(P_{j i}^{\star}(C)-P_{i j}^{\star}(C)\right)\right) .
$$

Notice that we use the Expected Shortfall as we are considering the situation of a single insurer.

Expressions (3.5) and (3.7) are fundamental. They describe the economics of proportional risk transfer and related capital requirements at the level of individual insurer $i$ as well as for the network itself. In line with (2.6), the overall amount of capital held by all insurance companies part of the network to cover their capital requirements is

$$
\operatorname{RBC}_{\text {Network }}^{\star}(C)=\sum_{i=1}^{n} \operatorname{RBC}\left(Z_{i}^{\star}(C)\right) .
$$

Furthermore,

$$
\mathbb{E}\left[Z_{\text {Network }}^{\star}(C)\right]=\sum_{i=1}^{n} \mathbb{E}\left[Z_{i}^{\star}(C)\right]
$$

is the total expected net underwriting profit generated by all insurers part of the network.

\section{Optimising risk transfer}

Risk transfer affects the profitability of risk exchanging parties as demonstrated in (3.1) and it also impacts the overall profitability of the network in (3.8). This gives rise to a series of questions. Can risk transfer be optimised such that it benefits all risk exchanging parties and the network overall? Will all parties be able to maximise their profitability through risk transfer? Is there a unique "optimal" or "fair" solution? 


\subsection{The network perspective}

There are in principle two different approaches to investigate the questions from above. The first approach relies on agent-based models where risk pricing plays the role of a utility function. The second approach depends on network-based models that describe dependencies and interactions between market participants from a more global point of view. We take the latter perspective and start our analysis from the expressions (3.5) and (3.7). Consider the maximisation problem of the profitability of the network $\mathbb{E}\left[Z_{\text {Network }}^{\star}(C)\right]$ with respect to $C \in[0,1]^{n \times n}$,

$$
\max _{C \in[0,1]^{n \times n}} \mathbb{E}\left[Z_{\text {Network }}^{\star}(C)\right] .
$$

This maximisation problem can be simplified by observing that the overall amount of premiums paid for risk transfer amongst the insurers disappears. The reason for this is that the network is closed, that is, any premium paid by an insurer will be received by another. Furthermore, we notice that the aggregate total loss of all insurers after risk transfer is equal to the overall gross loss.

Lemma 4.1. The total net underwriting profit generated by all insurers in the network is given by

$$
\sum_{i=1}^{n} Z_{i}^{\star}(C)=\sum_{i=1}^{n} P_{i}-L_{i}-\eta \operatorname{RBC}\left(Z_{i}^{\star}(C)\right) .
$$

Furthermore, the sum over the risk-based capital is given by

$$
\sum_{i=1}^{n} \operatorname{RBC}\left(Z_{i}^{\star}(C)\right)=\frac{1}{1-\eta} \sum_{i=1}^{n} \operatorname{ES}_{\alpha}\left(L_{i}^{\star}(C)\right)-P_{i} .
$$

Proof. We start with the sum over $Z_{i}^{\star}(C)$ as given in Equation (3.5). For the sake of argument define $P_{i i}^{\star}=0$ and add $\sum_{i=1}^{n} P_{i i}^{\star}-P_{i i}^{\star}=0$ to the sum. We immediately see that

$$
\sum_{i=1}^{n} \sum_{j \neq i}\left(P_{j i}^{\star}(C)-P_{i j}^{\star}(C)\right)=\sum_{i=1}^{n} \sum_{j=1}^{n}\left(P_{j i}^{\star}(C)-P_{i j}^{\star}(C)\right)=0 .
$$

For the loss we start with Equation (3.6), exchange the sums, and use the fact that for all $j \in\{1, \ldots, n\}$ we have $\sum_{i=1}^{n} C_{i j}=1$. We get

$$
\sum_{i=1}^{n} L_{i}^{\star}(C)=\sum_{i=1}^{n} \sum_{j=1}^{n} C_{i j} L_{j}=\sum_{j=1}^{n} L_{j} \sum_{i=1}^{n} C_{i j}=\sum_{j=1}^{n} L_{j} .
$$

The last assertion follows from Equation (3.7) and the translation property of the Expected Shortfall together with Equation (4.2).

With help of Lemma 4.1 we can now restate the maximisation problem of $\mathbb{E}\left[Z_{\text {Network }}^{\star}(C)\right]$ as a minimisation problem of the overall risk-based capital $\mathrm{RBC}_{\text {Network }}^{\star}(C)$. Indeed, we 
get

$$
\begin{aligned}
\max _{C \in[0,1]^{n \times n}} \mathbb{E}\left[Z_{\text {Network }}^{\star}(C)\right] & =\max _{C \in[0,1]^{n \times n}}\left\{\sum_{i=1}^{n} P_{i}-\mathbb{E}\left[L_{i}\right]-\eta \operatorname{RBC}\left(Z_{i}^{\star}(C)\right)\right\} \\
& =\sum_{i=1}^{n} P_{i}-\mathbb{E}\left[L_{i}\right]-\eta \min _{C \in[0,1]^{n \times n}} \operatorname{RBC}_{\text {Network }}^{\star}(C) .
\end{aligned}
$$

Hence, we can concentrate on the minimisation problem

$$
\min _{C \in[0,1]^{n \times n}} \operatorname{RBC}_{\text {Network }}^{\star}(C) .
$$

In analogy to Equation (2.6), $\mathrm{RBC}_{\text {Market }}$ is a lower bound to our minimisation problem. Using Lemma 4.1 together with subadditivity of the Expected Shortfall, we get

$$
\begin{aligned}
\operatorname{RBC}_{\text {Network }}^{\star}(C) & =\frac{1}{1-\eta} \sum_{i=1}^{n} \operatorname{ES}_{\alpha}\left(L_{i}^{\star}(C)\right)-P_{i} \\
& \geq \frac{1}{1-\eta}\left(\operatorname{ES}_{\alpha}\left(\sum_{i=1}^{n} L_{i}\right)-\sum_{i=1}^{n} P_{i}\right)=\mathrm{RBC}_{\text {Market }}
\end{aligned}
$$

In terms of the optimisation in (4.3), the question can be restated. How should the risk be shared amongst the insurers such that $\operatorname{RBC}_{\text {Network }}^{\star}(C)=\mathrm{RBC}_{\text {Market }}$ ? To answer this question, notice that the inequality in (4.4) can equivalently be stated as

$$
\sum_{i=1}^{n} \operatorname{ES}_{\alpha}\left(L_{i}^{\star}(C)\right)-\mathrm{ES}_{\alpha}\left(\sum_{i=1}^{n} L_{i}\right) \geq 0 .
$$

Therefore, any solution $C$ to the equation

$$
\sum_{i=1}^{n} \mathrm{ES}_{\alpha}\left(L_{i}^{\star}(C)\right)-\mathrm{ES}_{\alpha}\left(\sum_{i=1}^{n} L_{i}\right)=0
$$

is also a minimiser of $\operatorname{RBC}_{\text {Network }}^{\star}(C)$. The set of solutions to Equation (4.5) is given by

$$
\left\{C \in[0,1]^{n \times n} \mid \forall i, j \in\{1, \ldots, n\}: C_{i j}=C_{i i} \text { and } \sum_{i=1}^{n} C_{i j}=1\right\} .
$$

This can be seen from the following short calculation, where we use positive homogeneity of the Expected Shortfall and the fact that $\sum_{i=1}^{n} C_{i i}=\sum_{i=1}^{n} C_{i j}=1$, for any $j \in\{1, \ldots, n\}$,

$$
\begin{aligned}
\sum_{i=1}^{n} \mathrm{ES}_{\alpha}\left(L_{i}^{\star}(C)\right) & =\sum_{i=1}^{n} \mathrm{ES}_{\alpha}\left(\sum_{j=1}^{n} C_{i j} L_{j}\right)=\sum_{i=1}^{n} \mathrm{ES}_{\alpha}\left(\sum_{j=1}^{n} C_{i i} L_{j}\right) \\
& =\mathrm{ES}_{\alpha}\left(\sum_{j=1}^{n} L_{j}\right) \sum_{i=1}^{n} C_{i i}=\mathrm{ES}_{\alpha}\left(\sum_{j=1}^{n} L_{j}\right) .
\end{aligned}
$$


The set of solutions in (4.6) represents the first main result of this paper. It has a straightforward interpretation: the profitability at network level is optimised if after risk transfer all insurance companies hold a share of the market portfolio. In this situation, the sum of standalone risk-based capital corresponds to the overall riskbased capital of the market portfolio. This is a plausible and intuitive result. However, it should be kept in mind that it has been derived on the back of a consistent model of risk transfer realistically reflecting economics of capital costs.

Rewriting the set of optimal solutions in matrix form as in (3.4) yields

$$
C^{\mathrm{opt}}=\left(\begin{array}{cccc}
1-\sum_{j \neq 1}^{n} c_{j} & c_{1} & \cdots & c_{1} \\
c_{2} & 1-\sum_{j \neq 2}^{n} c_{j} & & c_{2} \\
\vdots & & \ddots & \vdots \\
c_{n} & \cdots & & 1-\sum_{j \neq n}^{n} c_{j}
\end{array}\right),
$$

where $c_{1}, \ldots, c_{n} \in[0,1]$ are constants such that $\sum_{i=1}^{n} c_{i}=1$. This is an equation with $n$ unknowns and consequently, there is no unique solution. An additional $n-1$ constraints will determine risk transfer unambiguously. From a practical perspective, the natural choice for the constraints is the capital available to support risk-based capital at the level of individual insurers. Such an approach reflects economic reality with limited capital available to support risk taking. Notice that following this approach, for one insurer the risk-based capital is determined implicitly. In economic terms, this insurer would need to adjust its capital base to ensure the overall network after risk transfer is adequately capitalised. This function of providing capital support to a network of insurance companies through risk transfer is typically performed by reinsurers. In Figure 1, this is illustrated by "I 4".

\subsection{Unique, fair and optimal solution}

As demonstrated in Section 4.1, there is no unique solution for the maximisation problem of the network without additional constraints, but rather a set of solutions. This raises the question whether one of these solutions can be regarded as "better" or "fair" towards all the insurers? In the following, we answer this question by applying principles of cooperative game theory to identify a unique fair solution for risk transfer. To this end, we follow the concept of fairness developed by M. Denault [12].

In more detail, Denault develops an approach to allocate risk-based capital between subportfolios of one single company. He considers coalitional games with fractional players for general coherent risk measures and identifies a unique fair allocation. We show that this fair allocation concept defines a unique, fair, and optimal risk transfer scheme $C^{\mathrm{f}}$ for a network of different insurance companies. In what follows, we will briefly explain the framework and demonstrate that the risk transfer scheme $C^{\mathrm{f}}$ results in an allocation of standalone risk-based capital that equals the Aumann-Shapley allocation. In the sense of Denault, this is considered fair and optimal.

Consider a coalitional game with fractional players $\left(N, \Lambda, R_{\rho}\right)$ consisting of

- a finite set $N=\{1, \ldots, n\} \subset \mathbb{N}$ of players with $|N|=n$, where in our case each player corresponds to a portfolio of an insurer, 
- a positive vector $\Lambda \in[0,1]^{n}$, where each element $\Lambda_{i}$ represents the maximal possible involvement of player $i \in N$, and

- a risk measure function $R_{\rho}:[0,1]^{n} \rightarrow \mathbb{R}$ with $\lambda \mapsto R_{\rho}(\lambda)$ such that $R_{\rho}(0)=0$.

So each player represents the portfolio of an insurer $i$ which is characterised by $Z_{i}$, the profit distribution. The vector $\Lambda \in[0,1]^{n}$ represents the maximal portion of each player's portfolio included in the coalition,

$$
Z=\sum_{i=1}^{n} \Lambda_{i} Z_{i}
$$

We introduce another vector $\lambda \in[0,1]^{n}, \lambda \leq \Lambda$, which defines the level of actual involvement of each player in the coalition. So the ratio $\frac{\lambda_{i}}{\Lambda_{i}}$ gives the proportion of the actual to the maximal involvement of player $i \in N$.

For fixed random variables $\left\{Z_{i}\right\}_{i \in\{1, . ., n\}}$, we define the risk measure function associated with a coherent risk measure $\rho$ as $R_{\rho}:[0,1]^{n} \rightarrow \mathbb{R}$ with

$$
R_{\rho}(\lambda)=\rho\left(-\sum_{i=1}^{n} \lambda_{i} Z_{i}\right) .
$$

Notice that $R_{\rho}(\Lambda)=\rho(-Z)$. The notion of coherency can be extended to these risk measure functions, see [12, Definition 11]. However, we will only need positive homogeneity, that is, for all $\gamma>0$ and $\lambda \in[0,1]^{n}$ we have $R_{\rho}(\gamma \lambda)=\gamma R_{\rho}(\lambda)$. This follows directly from the positive homogeneity of the coherent risk measure $\rho$.

Following Denault, we introduce the notion of fuzzy values. These are maps $\varphi$ which assign to each coalitional game with fractional players $\left(N, \Lambda, R_{\rho}\right)$ a unique per unit allocation vector,

$$
\varphi:\left(N, \Lambda, R_{\rho}\right) \mapsto\left(\varphi_{1}\left(N, \Lambda, R_{\rho}\right), \ldots, \varphi_{n}\left(N, \Lambda, R_{\rho}\right)\right)^{\top} \in \mathbb{R}^{n},
$$

such that

$$
\Lambda^{\top} \varphi\left(N, \Lambda, R_{\rho}\right)=R_{\rho}(\Lambda) .
$$

The concept of coherency is then further extended for fuzzy values and the implications for the fairness of these allocations are discussed. For the exact definition and the justification see [12, Definition 13] and the subsequent discussion.

A well known coherent fuzzy value is the Aumann-Shapley value. ${ }^{3}$ Given the existence of the partial derivative of $R_{\rho}$ in direction $\lambda_{i}, \partial_{\lambda_{i}} R_{\rho}$, it is for $i \in\{1, \ldots, n\}$ defined as

$$
\varphi_{i}^{\mathrm{AS}}\left(N, \Lambda, R_{\rho}\right)=\int_{0}^{1} \partial_{\lambda_{i}} R_{\rho}(\gamma \Lambda) \mathrm{d} \gamma .
$$

So the per unit allocation is the mean of the marginal risk of the $i$-th insurer as the involvement of all insurers increases uniformly from 0 to 1 . Using the fact that for a

\footnotetext{
${ }^{3}$ For a proof of the coherency of the Aumann-Shapley value see [12, Corollary 1].
} 
differentiable, $k$-homogeneous function $f: \mathbb{R}^{n} \rightarrow \mathbb{R}$, that means, for all $\gamma>0, x \in \mathbb{R}^{n}$ we have $f(\gamma x)=\gamma^{k} f(x)$, any of its partial derivatives are $(k-1)$-homogeneous, we can simplify the integral from above. Since the risk measure function $R_{\rho}$ is positively homogeneous, that means 1-homogeneous, we get

$$
\varphi_{i}^{\mathrm{AS}}\left(N, \Lambda, R_{\rho}\right)=\partial_{\lambda_{i}} R_{\rho}(\Lambda) \int_{0}^{1} 1 \mathrm{~d} \gamma=\partial_{\lambda_{i}} R_{\rho}(\Lambda) .
$$

This corresponds to the Euler allocation principle. The resulting per portfolio allocation is the gradient of $R_{\rho}$ evaluated at the maximal presence level $\Lambda$,

$$
\varphi^{\mathrm{AS}}\left(N, \Lambda, R_{\rho}\right)=\nabla R_{\rho}(\Lambda) .
$$

This gradient is the Aumann-Shapley risk allocation. It refers to the amount of risk allocated to each portfolio when $\Lambda=[1, \ldots, 1]^{\top} \in \mathbb{R}^{n}$.

Hence, choosing $\Lambda=[1, \ldots, 1]^{\top} \in \mathbb{R}^{n}$ and the Expected Shortfall, $\rho=\mathrm{ES}_{\alpha}$, we get

$$
\operatorname{RBC}(Z)=\mathrm{ES}_{\alpha}(-Z)=R_{\mathrm{ES}_{\alpha}}(\Lambda) .
$$

Therefore, the resulting risk capital allocation is

$$
\operatorname{RBC}_{Z}\left(Z_{i}\right)=\varphi_{i}^{\mathrm{AS}}\left(N, \Lambda, R_{\mathrm{ES} \alpha}\right)=\partial_{\lambda_{i}} R_{\mathrm{ES}_{\alpha}}(\Lambda)=\left.\partial_{\lambda_{i}} \mathrm{ES}_{\alpha}\left(-\sum_{i=1}^{n} \lambda_{i} Z_{i}\right)\right|_{\lambda=\Lambda}
$$

This expression can be simplified. Indeed, the Aumann-Shapley allocation with the Expected Shortfall as the risk measure corresponds to the allocation scheme based on the Conditional Expected Shortfall.

Proposition 4.2. For $i \in\{1, \ldots, n\}$ we have

$$
\partial_{\lambda_{i}} \operatorname{ES}_{\alpha}\left(-\sum_{i=1}^{n} \lambda_{i} Z_{i}\right)=\mathrm{CES}_{\alpha,-\sum_{i=1}^{n} \lambda_{i} Z_{i}}\left(-Z_{i}\right) .
$$

Proof. A proof is given in [25, Proposition 2.1, Lemma A.1]. Compare also D. Tasche [26].

Using Proposition 4.2 and Equation (4.7) with $\Lambda=(1, \ldots, 1)^{\top} \in \mathbb{R}^{n}$ yields

$$
\operatorname{RBC}_{Z}\left(Z_{i}\right)=\mathrm{CES}_{\alpha,-Z}\left(-Z_{i}\right)
$$

Following Section 4.1, the maximisers of the optimisation in (4.1) are the solutions of Equation (4.5) and only depend on losses before risk transfer, $L_{i}$, and after risk transfer, $L_{i}^{\star}$. Thus, let the $n$ insurers be represented by their gross loss amounts $L_{i}$ and the gross loss amount of the grand coalition be represented by the network itself. Using the Expected Shortfall as our risk measure, the overall risk of the network is quantified by

$$
\mathrm{ES}_{\alpha}\left(L_{\text {Network }}\right)=\mathrm{ES}_{\alpha}\left(\sum_{i=1} L_{i}\right)
$$


Assuming again $\Lambda=(1, \ldots, 1)^{\top} \in \mathbb{R}^{n}$, the resulting Aumann Shapley allocation,

$$
\varphi_{i}^{\mathrm{AS}}\left(N, \Lambda, R_{\mathrm{ES}_{\alpha}}\right)=\mathrm{CES}_{\alpha, L_{\text {Network }}}\left(L_{i}\right),
$$

is the fair risk allocation to insurer $i$. From Section 4.1 we know that the optimal risk transfer $C^{\mathrm{f}}$ belongs to the set

$$
\left\{C \in[0,1]^{n \times n} \mid \forall i, j \in\{1, \ldots, n\}: C_{i j}=C_{i i} \text { and } \sum_{i=1}^{n} C_{i j}=1\right\},
$$

and it is fair if the retained risk $L_{i}^{\star}$ after risk transfer equals the fair risk allocation in (4.8). Therefore, the optimal and fair risk transfer $C^{\mathrm{f}}$ is determined by the solution of the equation

$$
C_{i i}^{\mathrm{f}} \operatorname{ES}_{\alpha}\left(L_{\text {Network }}\right)=\operatorname{CES}_{\alpha, L_{\text {Network }}}\left(L_{i}\right),
$$

for all $i \in\{1, \ldots, n\}$. Hence, we have

$$
C_{i i}^{\mathrm{f}}=\frac{\operatorname{CES}_{\alpha, L_{\mathrm{Network}}}\left(L_{i}\right)}{\mathrm{ES}_{\alpha}\left(L_{\text {Network }}\right)},
$$

where both $\operatorname{CES}_{\alpha, L_{\text {Network }}}\left(L_{i}\right)$ and $\operatorname{ES}_{\alpha}\left(L_{\text {Network }}\right)$ are assumed to be positive. Equation (4.9) is the second main result of this paper. It defines a unique, fair and optimal solution for the risk transfer $C^{\mathrm{f}}$ across the network. This solution can be understood as defining risk transfer such that the diversification present at network level is effectively and efficiently allocated in a fair, optimal and unique way to individual insurers. As such the solution ensures that the positive impact of diversification on economic profitability is realised at the level of individual insurers in a fair and optimal way.

\section{Impact of risk transfer on systemic risk}

The network approach to risk transfer developed in this paper lends itself naturally to an analysis of systemic risk. Our framework can shed light on a range of aspects of systemic risk, including interconnectedness, complexity, and resilience at the level of individual insurance companies and the network itself. While a subsequent paper will analyse systemic risk in more detail, here we just highlight three critical aspects directly linked to risk transfer, sharing risk and diversification.

- Firstly, we notice that sharing risk and diversification through risk transfer will distribute risk across the network and thereby improve resilience at the level of individual insurers.

- On the other hand, we observe that risk transfer is increasing interconnectedness and may thus contribute to systemic risk itself.

- Lastly, we note that the optimisation of risk transfer through increasing diversification at the level of individual insurers may lead to a reduction of overall capital requirements. 


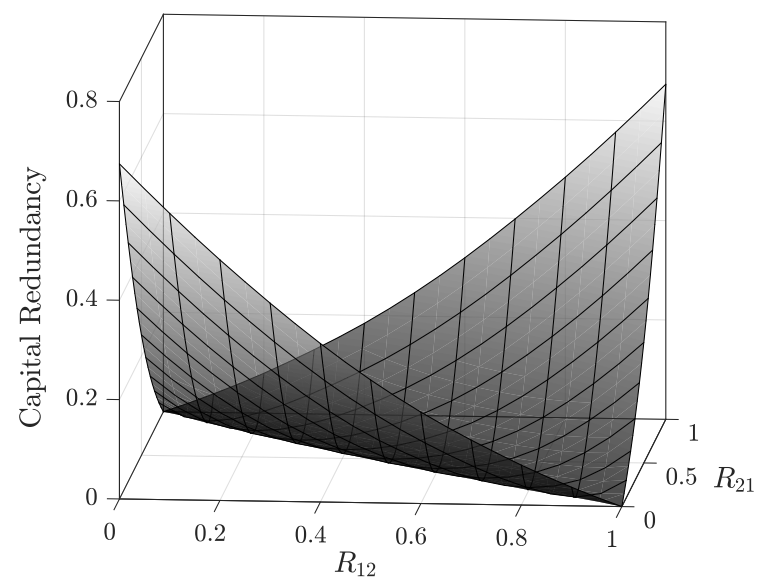

Figure 2: The graph visualises capital redundancy as defined by Equation (5.1). The $R_{12}$ and $R_{21}$ axes represent the level of risk retention of the first and second insurer, respectively.

The impact of optimisation of risk transfer on capital requirements at network level as compared to the capital requirement at market level is illustrated best by Equation (2.6). The ratio

$$
\frac{\mathrm{RBC}_{\text {Network }}-\mathrm{RBC}_{\text {Market }}}{\mathrm{RBC}_{\text {Market }}}
$$

describes how close the network is to optimal risk transfer. At the same time it also describes capital redundancy of the network relative to the market. See Figure 2 for a visual illustration. The graph illustrates Equation (5.1) as a function of different risk retention levels. For this we simulated the losses of two insurers. We assume that the losses have the same log-normal distribution and that they are independent. For each retention level pair we evaluate Equation (5.1). One can clearly see that the optimal risk retention pairs lie on the diagonal line connecting the points $(0,1)$ and $(1,0)$ on the $x y$-plane, as predicted in Section 4. In contrast, if both insurers either do not participate in risk transfer, point $(1,1)$, or decide to just swap their losses, point $(0,0)$, the network is furthest away from the optimum.

Optimising risk transfer reduces the ratio which means overall capital remaining at network level is reduced. From the point of systemic risk this may indicate that through optimising risk transfer, systemic risk is increased as the overall capital available at network level to cope with a systemic stress event is reduced.

Optimisation of risk transfer on the other hand leads to each insurance company of the network holding a fraction of the total market portfolio. Risk, including systemic risk, is shared evenly across all insurance companies preventing concentration of systemic risk at level of a single insurance company thereby increasing resilience. However, risk transfer is increasing interconnectedness. As a result, a systemic event through optimised risk transfer can impact the whole network thereby increasing systemic risk. Further analysis is needed to understand in more detail these competing effects of optimised risk transfer on systemic risk exposure and resilience at network level. Such an analysis will have to distinguish between idiosyncratic risk and systemic risk factors and their role in the optimisation of risk transfer through optimally sharing diversification. 


\section{Conclusions and outlook}

Risk transfer is a risk and capital management tool which enables insurers to meet capital and risk appetite constraints. At the same time, risk transfer is also a tool to enhance performance as it can balance portfolios and thereby improve risk-return characteristics. For a single insurer, optimising risk transfer essentially means tradingoff the cost of risk transfer to mitigated loss potential and reduced capital costs.

For a network of insurers, optimising risk transfer is less evident as optimisation can take different perspectives. To analyse this we considered risk transfer in the context of a network of insurance companies. Prompted by supervisory frameworks as, for example, Solvency II or SST requiring insurers to quantify risk-based capital using prescribed modelling principles and risk measures, a common, realistic, and economical premium principle is applied. Assuming an equal risk appetite and capital cost for all insurers, there is, in general, no solution which maximises the profitability of all insurance companies in the network. However, there is a set of solutions which maximise the overall profitability at the network level. These solutions are characterised by risk transfer such that all insurers hold a proportion of the market portfolio. The analysis also demonstrates that additional constraints will determine risk transfer unambiguously.

Absent of additional constraints cooperative game theory defines a unique, fair and optimal solution related to coherent risk allocation principles based on the Aumann Shapley value developed in [12]. This solution defines risk transfer such that the diversification present at network level is effectively and efficiently allocated in a fair, optimal, and unique way to individual insurers.

In practice, the fair and optimal solution defined by the Aumann Shapley value can be realised closest for insurance groups. Reason for this is that for insurance groups there exists an overarching rationale to optimise profitability not only at the level of individual insurance companies, but at the level of the insurance group itself. Thus, insurers which are part of the group are directly incentivised to form coalitions in terms of risk transfer that optimises profitability at the group level. The solution defined by the Aumann Shapley value not only optimises profitability, but also is fair in the sense that it recognises the contribution to group level diversification and through risk transfer efficiently allocates it back to individual insurers. It should be noted that the realisation of group level diversification through risk transfer instruments is the only way to recognise diversification under supervisory frameworks building on risk-based capital quantification. In this context, the interplay of optimal ownership structure and optimal risk transfer remains a topic for further analysis.

A related topic for further research is the impact of risk transfer on systemic risk. The network approach to risk transfer developed in this paper sheds light on a wide range of aspects of systemic risk, including in particular interconnectedness, complexity, and resilience at the level of individual insurance companies and the network itself. There are two critical facets to this. Firstly, sharing risk and diversification through risk transfer will distribute risk across the network and thereby improve resilience at the level of individual insurers. Risk transfer, on the other hand, is increasing interconnectedness and may thus contribute to systemic risk itself. The optimisation of risk transfer discussed in this paper may lead to a reduction of overall capital supporting risk and thus increase systemic risk exposure accordingly. Connecting concepts developed in the present paper with related research like [2] and [13] is a possible starting point to 
further investigate these and related questions.

\section{A Appendices}

\section{A.1 Proofs of Section 2}

The following lemmas show that with the choices in (2.4) and (2.5) our requirements described in Section 2.3 are satisfied. In analogy to Lemma 2.1, we get the following result.

Lemma A.1. With the choices in (2.4) and (2.5) we get

$$
\operatorname{RBC}_{Z}\left(Z_{i}\right)=\frac{\mathrm{CES}_{\alpha,-Z}\left(-U_{i}\right)}{1-\eta} .
$$

Proof. Using Equation (2.4), Equation (2.5), and the linearity of the conditional expectation, we get

$$
\operatorname{RBC}_{Z}\left(Z_{i}\right)=\mathrm{CES}_{\alpha,-Z}\left(-U_{i}+\eta \mathrm{RBC}_{Z}\left(Z_{i}\right)\right)=\mathrm{CES}_{\alpha,-Z}\left(-U_{i}\right)+\eta \mathrm{RBC}_{Z}\left(Z_{i}\right) .
$$

Solving for $\mathrm{RBC}_{Z}\left(Z_{i}\right)$ yields the result.

Furthermore, the Conditional Expected Shortfall defines an additive risk-based capital.

Lemma A.2. With the choices in (2.4) and (2.5) we get

$$
\sum_{i=1}^{n} \operatorname{RBC}_{Z}\left(Z_{i}\right)=\operatorname{RBC}(Z)
$$

Proof. Applying Lemma A.1 in the first equality and using the linearity of the conditional expectation in the second equality, we get

$$
\sum_{i=1}^{n} \operatorname{RBC}_{Z}\left(Z_{i}\right)=\frac{1}{1-\eta} \sum_{i=1}^{n} \operatorname{CES}_{\alpha,-Z}\left(-U_{i}\right)=\frac{1}{1-\eta} \operatorname{CES}_{\alpha,-Z}(-U) .
$$

Notice that by cash-additivity of the Value at Risk and Lemma 2.1 the condition $-Z \geq \operatorname{VaR}_{\alpha}(-Z)$ is equivalent to $-U \geq \operatorname{VaR}_{\alpha}(-U)$. Hence,

$$
\mathrm{CES}_{\alpha,-Z}(-U)=\mathrm{CES}_{\alpha,-U}(-U)=\mathrm{ES}_{\alpha}(-U) .
$$

Applying Lemma A.1 again yields the result.

Acknowledgements We gratefully acknowledge the careful review of a preliminary version of this work and the constructive feedback provided by an anonymous referee. 


\section{References}

[1] Acerbi, C., and Tasche, D. On the coherence of expected shortfall. Journal of Banking \& Finance 26, 7 (2002), 1487 - 1503.

[2] Acharya, V. V., Pedersen, L. H., Philippon, T., and Richardson, M. Measuring Systemic Risk. The Review of Financial Studies 30, 1 (2016), 2-47.

[3] Artzner, P., Delbaen, F., Eber, J.-M., and Heath, D. Coherent Measures of Risk. Mathematical Finance 9, 3 (1999), 203-228.

[4] Asimit, A., Badescu, A., and Tsanakas, A. Optimal risk transfers in insurance groups. European Actuarial Journal 3 (2013), 159-190.

[5] Asimit, V., Badescu, A., Haberman, S., and Kim, E.-S. Efficient risk allocation within a non-life insurance group under solvency ii regime. SSRN Electronic Journal (01 2015).

[6] Biagini, F., Doldi, A., Fouque, J.-P., Frittelli, M., and Meyer-Brandis, T. Systemic optimal risk transfer equilibrium. https://arxiv.org/abs/1907.04257v1, 2019. Accessed July 15, 2019.

[7] Centeno, M. d. L. Measuring the effects of reinsurance by the adjustment coefficient in the sparre anderson model. Insurance: Mathematics and Economics 30 (02 2002), 37-49.

[8] Dacorogna, M. A change of paradigm for the insurance industry. Annals of Actuarial Science 12 (2018), 211-232.

[9] Daykin, C., Pentikainen, T., and Pesonen, M. Practical Risk Theory for Actuaries. New York: Chapman and Hall, 1993.

[10] de Finetti, B. Il problema dei pieni, vol. 11. 1940, pp. 1-88.

[11] Delbaen, F. Coherent Risk Measures on General Probability Spaces. Springer Berlin Heidelberg, Berlin, Heidelberg, 2002, pp. 1-37.

[12] Denault, M. Coherent allocation of risk capital. Journal of Risk 4 (1999), 1-34.

[13] Engle, R. F., and Brownlees, C. SRISK: A conditional capital shortfall measure of systemic risk. The Review of Financial Studies 30, 1 (2016), 48-79.

[14] Ettlin, N. Optimal risk sharing: An analysis of risk-based capital allocation and risk transfer in the insurance industry, 2018. Master's Thesis, University of Basel.

[15] Haier, A., Molchanov, I., and Schmutz, M. Intragroup transfers, intragroup diversification and their risk assessment. Annals of Finance 12 (2016), 363-392.

[16] Hamm, A.-M., Knispel, T., and Weber, S. Optimal risk sharing in insurance networks - an application to asset-liability management. https://www.stochastik. uni-hannover.de/fileadmin/institut/pdf/ORS_20190323.pdf, 2019. Accessed July 15, 2019. 
[17] Kaluszka, M. Optimal reinsurance under mean-variance premium principles. Insurance: Mathematics and Economics 28 (02 2001), 61-67.

[18] Keller, P. Group diversification. The Geneva Papers on Risk and Insurance Issues and Practice 32 (2007), 382-392.

[19] KPMG. Cost of capital study 2019. https://assets.kpmg/content/dam/kpmg/ ch/pdf/cost-of-capital-study-2019.pdf, 2019. Accessed April 15, 2020.

[20] Kull, A. Sharing risk - an economic perspective. ASTIN Bulletin 39, 2 (2009), 591-613.

[21] Kupper, M., and Filipović, D. On the group level swiss solvency test. $97-115$.

[22] Liebrich, F.-B., and Svindland, G. Risk sharing for capital requirements with multidimensional security markets. Finance and Stochastics 23, 4 (2019), 925973.

[23] Mayer, T., Kull, A., Keller, P., and Portmann, H. Risk Sharing in Insurance Groups. Risk Books, 2009.

[24] McNeil, A. J., Frey, R., and Embrechts, P. Quantitative Risk Management: Concepts, Techniques, and Tools. Princeton Series in Finance. Princeton University Press, 2005.

[25] Scaillet, O. Nonparametric estimation and sensitivity analysis of expected shortfall. Mathematical Finance: An International Journal of Mathematics, Statistics and Financial Economics 14, 1 (2004), 115-129.

[26] Tasche, D. Risk contributions and performance measurement. https://pdfs . semanticscholar.org/2659/60513755b26ada0b4fb688460e8334a409dd.pdf, 2000. Accessed March 30, 2018.

[27] Venter, G. G. Capital allocation survey with commentary. North American Actuarial Journal 8, 1 (2004), 96-107. 


\section{Swiss Finance Institute}

Swiss Finance Institute (SFI) is the national center for fundamental research, doctoral training, knowledge exchange, and continuing education in the fields of banking and finance. SFI's mission is to grow knowledge capital for the Swiss financial marketplace. Created in 2006 as a public-private partnership, SFI is a common initiative of the Swiss finance industry, leading Swiss universities, and the Swiss Confederation.

\section{swiss : finance: institute}

c/o University of Geneva, Bd. Du Pont d'Arve 42, CH-1211 Geneva 4 T+41223798471,rps@sfi.ch,www.sfi.ch 